national Journal of Biological Macromolecules (IJBM) and Enzyme and Microbial Technology (EMT) come under the imprint of a publishing house more famous for women's magazines than science journals.

The object of IJBM is to publish articles on all aspects of the structure, function and interactions of biological macromolecules and their assemblies. Contributions appear in the form of original research papers, preliminary communications and "Notes to the Editor", as well as in a correspondence column. There is little doubt that the various studies covered by the umbrella title of "biological macromolecules" would benefit from cross-fertilization, but I wonder whether there is enough cohesion in papers covering carbohydrates, nucleic acids and proteins to attract contributors (and subscribers) in the face of competition from the more specialist journals.

EMT is also interdisciplinary in aim, and beautifully produced in a large format. The journal was launched to provide a forum for the coordination of biotechnology and bioaffinity, especially the directed use of enzymes and microorganisms. Research papers, short papers and review articles are accepted. The growing importance of this field is unquestionable, but it involves many scientists and engineers from diverse backgrounds. Only now are they learning how to work together, and EMT should help them to talk to each other. The journal is certainly timely and I understand that it is proving to be a very successful venture.

Providing channels of communication between isolated fields is evidently seen by publishers as a boom area for the 1980s. Yet another interdisciplinary journal is Biological Trace Element Research (BTER), the aim of which is to emphasize the integrative aspects of trace metal research in all appropriate fields. The journal publishes regular papers and reviews with a reasonable time-lag of about five months. Undeniably, this is an expanding area of research and one which calls for papers that are written with a wide audience in mind. That this is not always achieved is best exemplified by finding papers entitled "Epidemiological Study of Chromium Platers in Japan" and "Chromate Metabolism in Liver Microsomes" virtually cheek by jowl in the journal. Nonetheless, BTER is a wellproduced journal that may fulfil a useful role in a developing area of toxicology.

Also well produced and from Humana Press comes Cell Biophysics (CB). The journal carries original papers, and occasional reviews on the key experimental, methodological and theoretical investigations on cells and their principal organelles and subfragments by biophysical methods. The aim is to publish studies at the cellular rather than the biochemical level.

While I can appreciate the general objective, in that cell biology is of much current interest now that we have such excellent means for examining cellular events, 1 would not have thought that the inclusion of "biophysics" would be an attractive feature. In my terms, $C B$ is not a specialist journal, for the range of subject matter is so broad that each issue needs to contain many papers if it is to attract, and hold, attention. Thus far CB has not published a sufficient amount of material to make good this inherent drawback in its concept.

The scope of the Journal of Biochemical and Biophysical Methods (JBBM) is, in its different way, as broad as that of CB. Yet I would guess that the future of this journal is assured from the contributions' side there is generally no dearth of papers for a methods' journal. The role that JBBM hopes to take is simply expressed in its title, and the journal publishes regular papers, short notes, short reviews and book reviews. Publication is fairly rapid, some five months after acceptance.

I should confess a bias against methods' journals. Biochemists do not specialize in techniques per se but in areas of science; at the same time, although rarely exciting, methods do of course provide the backbone to experimental research. My

biochemical and

biophysical

methods

doubt about JBBM lies in the fact that the papers in the four issues available to me in the main report modifications of existing practice. One would have thought that to thrive, rather than merely survive, the journal needs to poach genuine "breakthrough" papers from the large general or specialist journals.

An initial (and vital) step in the launching of a new journal is the gathering of names for the editorial board. In part this is an exercise in window dressing - convincing the potential subscriber that the journal has a standing by association with the renowned in his field. In common with most of the journals covered here, Membrane Biochemistry (MB) has assembled an impressive board of some 40 scientists of widely scattered affiliations. An increasing number of biochemists and biophysicists regard themselves as membraneologists, so here we have a journal which should satisfy a reasonably homogeneous group of readers. MB publishes straightforward research papers but there is no mention of dates of acceptance, so speed of publication cannot be gauged.

This area of science is popular and there are plenty of papers to fill the pages of its journals. However MB will have fierce competition for subscribers, and, although well produced, it is not a cost-effective pro-

\section{New Journals' Issue}

THE brief given to the authors in this issue was to limit themselves to comment on the journals sent to them for review. Reviewers were asked to avoid detailed discussion of general questions of periodical publishing and information systems. In the following pages, then, will be found description, comparison and criticism of some 120 journals, but little consideration of the wider aspects of the primary literature.

The criteria used to decide whether or not a given publication should be included in this issue were that:

(i) the first number appeared, or the journal was re-titled, between January 1978 and May 1980 (a few exceptions have been made to this rule);

(ii) it is published at least three times a year;

(iii) the main language used is English.

Thus, broadly, periodicals of professional interest to scientists were considered for review, with the exception of abstracts' journals. The cut-off date of May 1980 was chosen to allow enough issues of a journal to have been published, so as to give reviewers a reasonable sample of material on which to base a judgement.

To many (though probably not publishers, subscription agents and librarians), the number of new titles appearing over this period may come as a surprise. Yet many journals submitted for review are not covered here. Some of these are doubtless worthy publications, and their omission implies no prejudgement of their value. A further consequence of constraints on space in this issue is that the shortest possible (unconventional) title abbreviations have been used in the reviews.

Where possible, reviewers were asked to cover a number of broadly related journals, but in many instances this was impracticable. The preponderance of titles in the biological sciences reflects the bias of the material sent in for review and, presumably, a current trend in journal publishing.

Publishers and journal sponsors were requested to submit four issues - the first, the most recent and two others of each title for review (in some cases fewer were available). Opinions expressed in the reviews are not based on a full back-run of a journal, but on a sample, and apply to mid-1981 at the latest. The details of editors and frequency of publication, and the basic annual subscription prices appearing at the top of each article are given, where possible, for 1982. These details are taken from information supplied by publishers or from back issues, and are not necessarily complete. Readers should check prices, in particular, with the publisher concerned.

An index to the journals reviewed appears on p.369. 